\title{
The higher rank numerical range of nonnegative matrices
}

\author{
Aik. Aretaki and J. Maroulas*
}

April 20, 2022

\begin{abstract}
In this article the well known "Perron-Frobenius theory" is investigated involving the higher rank numerical range $\Lambda_{k}(A)$ of an irreducible and entrywise nonnegative matrix $A$ and extending the notion of elements of maximum modulus in $\Lambda_{k}(A)$. Further, an application of this theory to the $\Lambda_{k}(L(\lambda))$ of a Perron polynomial $L(\lambda)$ is elaborated via its companion matrix $C_{L}$.
\end{abstract}

Key words:Perron-Frobenius theory, nonnegative matrix, Perron polynomial, higher rank numerical range, rank-k numerical radius.

AMS Subject Classifications: 15B48, 15A60, 47A12.

\section{Introduction}

Let $\mathcal{M}_{n}(\mathbb{C})$ be the algebra of matrices $A=\left[a_{i j}\right]_{i, j=1}^{n}$ with entries $a_{i j} \in \mathbb{C}$ and $k \geq 1$ be a positive integer. The k-rank numerical range $\Lambda_{k}(A)$ of a matrix $A \in \mathcal{M}_{n}$ is defined by

$$
\Lambda_{k}(A)=\left\{\lambda \in \mathbb{C}: P A P=\lambda P \text { for some } P \in \mathcal{P}_{k}\right\},
$$

where $\mathcal{P}_{k}$ is the set of all orthogonal projections $P$ of $\mathbb{C}^{n}$ onto any $k$ dimensional subspace $\mathcal{K}$ of $\mathbb{C}^{n}$. Equivalently,

$$
\Lambda_{k}(A)=\left\{\lambda \in \mathbb{C}: X^{*} A X=\lambda I_{k}, X \in \mathcal{M}_{n, k}(\mathbb{C}), X^{*} X=I_{k}\right\} .
$$

For any $k$, the sets $\Lambda_{k}(A)$ are generally called higher rank numerical range. The concept of higher rank numerical range has been introduced by Choi et al in [2, 3, 4, 5] and studied thoroughly by other researchers in [11, 12, 17. Apparently, for $k=1, \Lambda_{k}(A)$ yields the classical numerical range of a matrix $A$ [7, 9], i.e.

$$
\Lambda_{1}(A) \equiv F(A)=\left\{x^{*} A x: x \in \mathbb{C}^{n}, x^{*} x=1\right\}
$$

${ }^{*}$ Department of Mathematics, National Technical University of Athens, Zografou Campus, Athens 15780, Greece. E-mail address: maroulas@math.ntua.gr. 
and it is readily verified

$$
F(A) \supseteq \Lambda_{2}(A) \supseteq \ldots \supseteq \Lambda_{k}(A) .
$$

Moreover, the notion of the numerical radius for the numerical range,

$$
r(A)=\max \{|z|: z \in F(A)\},
$$

has been extended to the rank $k$-numerical radius

$$
r_{k}(A)=\max \left\{|z|: z \in \Lambda_{k}(A)\right\},
$$

considering $r_{k}(A)=-\infty$, whenever $\Lambda_{k}(A)$ is an empty set [11]. Note that $r(A) \geq r_{k}(A)$ and $r(A) \geq \rho(A)$, where $\rho(\cdot)$ denotes the spectral radius of a matrix, i.e. $\rho(\cdot)=\max \{|\lambda|: \lambda \in \sigma(\cdot)\}$, with $\sigma(\cdot)$ to be the spectrum of a matrix.

We mention that an $n \times n$ matrix $A$ is said to be nonnegative when each $a_{i j} \geq 0$, and this is denoted by writing $A \geq 0$. Similarly, $A$ is said to be positive whenever each $a_{i j}>0$, denoted by $A>0$. The matrix $A \in \mathcal{M}_{n}$ is called reducible when there is a permutation matrix $P$ such that

$$
P^{T} A P=\left[\begin{array}{cc}
R & S \\
0 & T
\end{array}\right]
$$

where $R, T$ are both square. For $n=1$, should be $A=0$. Otherwise, $A$ is said to be irreducible. If $A$ is nonnegative and irreducible having $q>1$ eigenvalues of maximum modulus, then it is called imprimitive and $q$ is referred to as index of imprimitivity. In case $q=1, A$ is characterized as primitive.

It is well known that Perron-Frobenius theory concerns the spectral properties of positive and nonnegative matrices, namely the existence of positive or nonnegative eigenvalues and eigenvectors [8]. In addition, Issos' treatment contributes extensions of the Perron-Frobenius theorem to the numerical range of a nonnegative and irreducible matrix $A$, relating the $\rho(A)$ with the $r(A)$ [10. These results give the motivation for further investigation of $\Lambda_{k}(A)$ in the case of a nonnegative and irreducible matrix $A$ (section 2).

In the next section, we present applications of the Perron-Frobenius theory derived for a matrix polynomial, considering the higher rank numerical range of matrix polynomials.

\section{Nonnegative and irreducible matrices}

According to Issos' main theorems [10, Th.4,7], if the numerical range $F(A)$ of a nonnegative and irreducible matrix $A$ has $q$ maximal elements, then they 
are equally spaced around a circle centered at the origin through a constant angle, with one of them lying on the positive real axis. In particular,

$$
\mathcal{F}(A)=\left\{r(A) e^{\mathbf{i} \frac{2 \pi t}{q}}: t=0, \ldots, q-1\right\}
$$

is exactly the set of all the maximal elements in $F(A)$ and to the numerical radius $r(A) \in F(A)$ there always corresponds a positive unit vector. Furthermore, Issos also proved that the cardinality of the set $\mathcal{F}(A)$ coincides with the index of imprimitivity of $A$.

In this section, we investigate to what extend do Issos' results apply to $\Lambda_{k}(A)$ of a nonnegative and irreducible matrix $A$, taking into consideration that it is a non empty set [11]. At this point, we define the maximal elements in $\Lambda_{k}(A)$ to constitute the set:

$$
\mathcal{F}_{k}(A)=\left\{z \in \Lambda_{k}(A):|z|=r_{k}(A)\right\},
$$

which for $k=1$ is equal to $\mathcal{F}(A)$. Although the numerical range $F(A)$ of a nonnegative matrix $A$ always contains the numerical radius $r(A)>0$ [10, Th.1], it is not generally true that $r_{k}(A) \in \Lambda_{k}(A)$, as we may observe in the following example.

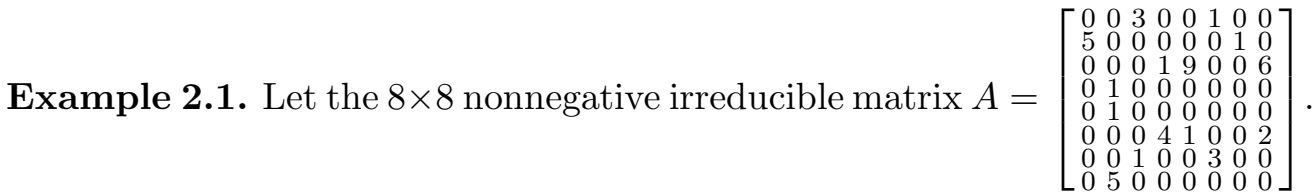

The outer curve in the following figure illustrates the boundary of $F(A)$, whereas the second and third inner curves illustrate the boundary of $\Lambda_{2}(A)$ and $\Lambda_{3}(A)$, respectively. Apparently, $0<r(A) \in F(A)$, but in the figure, we easily recognize that $0<r_{2}(A) \notin \Lambda_{2}(A)$ and $0<r_{3}(A) \notin \Lambda_{3}(A)$. Note that $A$ has 4 maximal eigenvalues, which are marked by "+" and $F(A)$ has 4 maximal elements, as well.

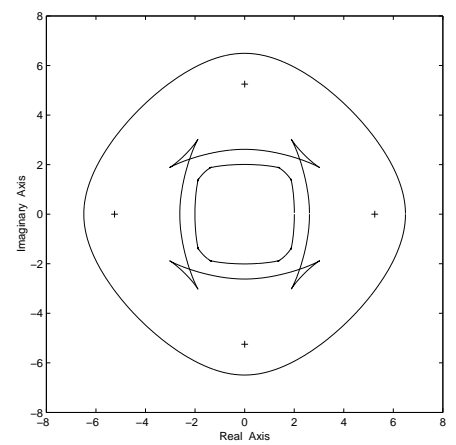

The following Lemma generalizes a familiar condition for rotational invariance and symmetry about the origin to the case of the higher rank numerical range. 
Lemma 2.2. Let $A \in \mathcal{M}_{n}(\mathbb{C})$ be permutation similar (hence unitarily similar) to the matrix

$$
C=\left[\begin{array}{ccccc}
0 & C_{12} & 0 & \cdot & 0 \\
0 & 0 & C_{23} & \cdot & 0 \\
\cdot & \cdot & \cdot & \cdot & \cdot \\
0 & \cdot & \cdot & 0 & C_{q-1, q} \\
C_{q 1} & 0 & \cdot & \cdot & 0
\end{array}\right]
$$

with the zero blocks along the main diagonal be square. For $j=1, \ldots, k$ such that $r_{j}(A) \neq\{-\infty, 0\}$, we have:

I. $\Lambda_{j}(A)=\Lambda_{j}\left(e^{i \frac{2 \pi t}{q}} A\right)$, for $t=0,1, \ldots, q-1$

II. $\mu \in \Lambda_{j}(A)$ if and only if $\mu e^{i \frac{2 \pi t}{q}} \in \Lambda_{j}(A)$, for $t=0,1, \ldots, q-1$

III. $\Lambda_{j}(A)$ is symmetric with respect to the origin, if $q=2 t$.

Proof. I. By the proof of Theorem 6 in [10], $D^{-1} C D=e^{\mathbf{i} \theta} C$, where $D=$ $I_{n_{1}} \oplus e^{\mathrm{i} \theta} I_{n_{2}} \oplus \ldots \oplus e^{\mathbf{i}(q-1) \theta} I_{n_{q}}$ is unitary diagonal with $\theta=\frac{2 \pi t}{q}$ and $n_{1}+\ldots+$ $n_{q}=n$, where $n_{l}(l=1, \ldots, q)$ is the dimension of the $l$-th diagonal block of $C$. Hence, $e^{\mathrm{i} \theta} A=\left(P^{T} D P\right)^{-1} A\left(P^{T} D P\right)$, where $P$ is the permutation matrix such that $A=P^{T} C P$, and $\Lambda_{j}(A)=\Lambda_{j}\left(e^{\mathbf{i} \theta} A\right)$, for any $j=1, \ldots, k$.

II. By statement (I), we have that

$$
\mu \in \Lambda_{j}(A) \Leftrightarrow e^{\mathbf{i} \frac{2 \pi t}{q}} \mu \in \Lambda_{j}\left(e^{\mathbf{i} \frac{2 \pi t}{q}} A\right)=\Lambda_{j}(A),
$$

for all $t=0,1, \ldots, q-1$ and $j=1, \ldots, k$.

III. Let $q=2 t$, then by (II), $\mu \in \Lambda_{j}(A)$ if and only if $e^{\mathrm{i} \frac{2 \pi t}{2 t}} \mu \in \Lambda_{j}(A)$, i.e. $e^{\mathbf{i} \pi} \mu=-\mu \in \Lambda_{j}(A)$ for $j=1, \ldots, k$.

Example 2.3. Let the matrix $A=\left[\begin{array}{cccc}0 & 0 & i & 0 \\ 0 & 0 & -i & 0 \\ 0 & 0 & 0 & 2 \\ 3+2 i & 1 & 0 & 0\end{array}\right]$ as in (2.1) with $q=3$. The boundary of $\Lambda_{2}(A)$ is illustrated below by the arched triangle. Apparently, $\Lambda_{2}(A)$ is rotationally invariant about the origin through an angle of $\frac{2 \pi t}{3}$ for $t=0,1,2$, confirming Lemma 2.2(I,II).

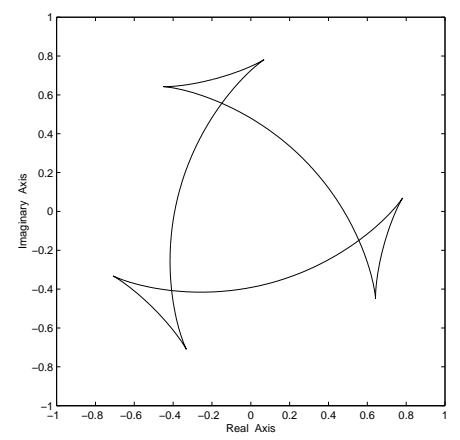


Any matrix $A$ being permutation similar to the matrix $C$ in (2.1) is said to be $q$-cyclic and the largest positive integer $q$ such that $A$ is $q$-cyclic is called the cyclic index of $A$.

Regarding the number of maximal elements in $\Lambda_{k}(A)$ and their location on the complex plane, we refer to the following result.

Proposition 2.4. Let $A \in \mathcal{M}_{n}(\mathbb{R})$ be imprimitive with index of imprimitivity $q>1$ such that $r_{k}(A)>0$. Then

$$
\mathcal{F}_{j}(A)=\left\{r_{j}(A) e^{i\left(\theta_{j}+\frac{2 \pi t}{q}\right)}: t=0, \ldots, q-1\right\},
$$

for every $j=1, \ldots, k$ with $\theta_{j}=0$ or $\theta_{j}=\frac{\pi}{q}$.

Proof. Since $q>1$ is the index of imprimitivity of $A$, there is a permutation matrix $P$ such that

$$
P^{T} A P=\left[\begin{array}{ccccc}
0 & C_{12} & 0 & \cdot & 0 \\
0 & 0 & C_{23} & \cdot & 0 \\
\cdot & \cdot & \cdot & \cdot & \cdot \\
0 & \cdot & \cdot & 0 & C_{q-1, q} \\
C_{q 1} & 0 & \cdot & \cdot & 0
\end{array}\right]
$$

Let $\theta_{j} \in[0,2 \pi)$ be the principal argument such that $0<r_{j}(A) \in \Lambda_{j}\left(e^{-\mathbf{i} \theta_{j}} A\right)$ with $j=1, \ldots, k$. Then, by Lemma $2.2(\mathrm{II})$, we have $r_{j}(A) e^{\mathbf{i}\left(\theta_{j}+\frac{2 \pi t}{q}\right)} \in \Lambda_{j}(A)$ for $t=0,1, \ldots, q-1$ and $j=1, \ldots, k$, whereupon we obtain

$$
\mathcal{F}_{j}(A) \supseteq\left\{r_{j}(A) e^{\mathbf{i}\left(\theta_{j}+\frac{2 \pi t}{q}\right)}: t=0, \ldots, q-1\right\}
$$

for every $j=1, \ldots, k$.

The index of imprimitivity $q$ is equal to the largest positive integer such that $A$ is unitarily diagonally similar to the matrix $e^{i \frac{2 \pi}{q}} A$, equivalently, the matrices $e^{-\mathbf{i} \theta_{j}} A$ and $e^{\mathbf{i}\left(-\theta_{j}+\frac{2 \pi}{q}\right)} A$ are unitarily diagonally similar for the largest positive integer q. Therefore, the set

$$
\left\{0, \frac{2 \pi}{q}, \ldots, \frac{2 \pi(q-1)}{q}\right\}
$$

is the cyclic group modulo $2 \pi$ of the largest order, concluding that there does not exist $\phi=\frac{2 \pi}{p}<\frac{2 \pi}{q}$ such that $r_{j}(A) e^{\mathbf{i} \phi} \in \Lambda_{j}\left(e^{-\mathbf{i} \theta_{j}} A\right)$ for $j=1, \ldots, k$. Hence we establish the equality

$$
\mathcal{F}_{j}(A)=\left\{r_{j}(A) e^{\mathbf{i}\left(\theta_{j}+\frac{2 \pi t}{q}\right)}: t=0, \ldots, q-1\right\} .
$$

In addition, if we denote by - the conjugate of a set, it is clear that $\Lambda_{j}(A)=\overline{\Lambda_{j}(A)}$, since $A \in \mathcal{M}_{n}(\mathbb{R})$, i.e. $\Lambda_{j}(A)$ is symmetric with respect to the real axis. Due to this symmetry and the equation (2.3), if we consider $\theta_{j} \neq 0$, we obtain $2 \pi-\theta_{j}=\theta_{j}+\frac{2 \pi(q-1)}{q}$. Hence, $\theta_{j}=\frac{\pi}{q}$ and the proof is complete. 
In view of the preceding proposition, the number of elements of maximum modulus in each $\Lambda_{j}(A)$ of a nonnegative and irreducible matrix $A$, for $j=$ $1,2, \ldots, k$, is equal to the index of imprimitivity $q$. Especially, they are all successively distributed around a circle centered at the origin through the constant angle of $\frac{2 \pi}{q}$. Furthermore, it is also observed that after a clockwise rotation of $\Lambda_{j}(A)$ about the origin through the angle of $\frac{\pi}{q}$, it is achieved $0<r_{j}(A) \in \Lambda_{j}\left(e^{-\mathbf{i} \frac{\pi}{q}} A\right)$.

On the other hand, if we consider the primitive class of nonnegative matrices $A(q=1)$, then the sets $\Lambda_{j}(A)$, for $j=2, \ldots, k$ do not necessarily have only one maximal element, contrary to the $F(A)$. This implication is justified by the symmetry of the sets $\Lambda_{j}(A)$ with respect to the real axis, when $r_{j}(A) e^{\mathbf{i} \theta_{j}} \in \Lambda_{j}(A)$ for some $\theta_{j} \in(0,2 \pi)$, then also $r_{j}(A) e^{-\mathbf{i} \theta_{j}} \in \Lambda_{j}(A)$.

Example 2.5. Let the $8 \times 8$ imprimitive matrix $A=\left[\begin{array}{llllllll}0 & 0 & 2 & 0 & 0 & 6 & 0 & 0 \\ 1 & 0 & 0 & 0 & 0 & 0 & 7 & 0 \\ 0 & 0 & 0 & 2 & 3 & 0 & 0 & 4 \\ 0 & 3 & 0 & 0 & 0 & 0 & 0 & 0 \\ 0 & 3 & 0 & 0 & 0 & 0 & 0 & 0 \\ 0 & 0 & 0 & 4 & 0 & 0 & 0 & 2 \\ 0 & 0 & 1 & 0 & 0 & 3 & 0 & 0 \\ 0 & 9 & 0 & 0 & 0 & 0 & 0 & 0\end{array}\right]$ with index of imprimitivity $q=4$. The boundaries of $F(A) \equiv \Lambda_{1}(A) \supseteq \Lambda_{2}(A) \supseteq$ $\Lambda_{3}(A)$ are illustrated below on the left figure and we verify that all $\Lambda_{j}(A)$ have $q=4$ maximal elements $(j=1,2,3)$, which are equally spaced through the angle of $t \frac{\pi}{2}, t=0,1,2,3$. Specifically, $r_{2}(A) e^{\mathbf{i} \frac{\pi}{4}} \in \Lambda_{2}(A), r_{3}(A) \in \Lambda_{3}(A)$. For the primitive case, let the nonnegative irreducible matrix $B=\left[\begin{array}{llllll}0 & 1 & 0 & 0 & 0 & 0 \\ 0 & 0 & 1 & 0 & 0 & 0 \\ 0 & 0 & 0 & 1 & 0 & 0 \\ 0 & 0 & 0 & 0 & 1 & 0 \\ 0 & 0 & 0 & 0 & 0 & 0 \\ 1 & 1 & 1 & 0 & 0 & 0\end{array}\right]$ with only one maximal eigenvalue. The boundaries of $F(B) \supseteq \Lambda_{2}(B)$ are illustrated on the right figure, revealing that $\Lambda_{2}(B)$ has two symmetric maximal elements, with respect to the negative real semi axis, whereas $F(B)$ has only one maximal element $r(B)$. The maximal eigenvalues of $A$ and $B$ are marked by "+" in both figures.
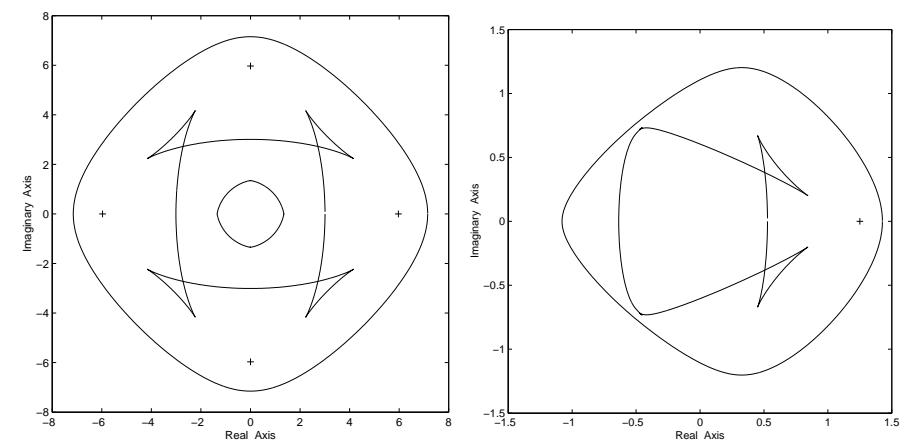

Remark 2.6. It is quite interesting to note that the $n$-cyclic permutation matrix $P_{n}=\left[\begin{array}{cc}0 & I_{n-1} \\ 1 & 0\end{array}\right] \in \mathcal{M}_{n}(\mathbb{R})$ is a special form of imprimitive matrix of index $n$, with spectrum the $n$-th roots of unity $z_{t}=e^{2 \pi t \mathbf{i} / n}, t=0, \ldots, n-1$. Apparently, $P_{n}$ is unitary and $F\left(P_{n}\right)=c o\left(\left\{z_{0}, z_{1}, \ldots, z_{n-1}\right\}\right)$, where $c o(\cdot)$ 
denotes the convex hull of a set. By corollary 2.8 in [6], whenever $2 k<n$, we have that

$$
\Lambda_{k}\left(P_{n}\right)=\operatorname{co}\left(\left\{\tilde{z}_{0}, \tilde{z}_{1}, \ldots, \tilde{z}_{n-1}\right\}\right),
$$

with $\tilde{z}_{t}(t=0, \ldots, n-1)$ to be intersection points of the line segments $\left[z_{t}, z_{t+k}\right]$ and $\left[z_{t+1}, z_{t+n-k+1}\right]$, when $z_{j}=z_{j-n}$ for $j>n-1$.

In addition, for the powers of $P_{n}$, we have that $\Lambda_{k}\left(P_{n}^{\alpha}\right)=\Lambda_{k}\left(P_{n}\right)(1 \leq \alpha \leq$ $n-1)$, since the matrices $P_{n}^{\alpha}$ are permutation similar to $P_{n}$. Also, $P_{n}^{n}=I_{n}$, whereupon $\Lambda_{k}\left(P_{n}^{n}\right)=\{1\}$.

For instance, $F\left(P_{5}\right)$ and $\Lambda_{2}\left(P_{5}\right)$ for the 5-cyclic permutation $P_{5}=\left[\begin{array}{lllll}0 & 1 & 0 & 0 & 0 \\ 0 & 0 & 1 & 0 & 0 \\ 0 & 0 & 0 & 1 & 0 \\ 0 & 0 & 0 & 0 & 1 \\ 1 & 0 & 0 & 0 & 0\end{array}\right]$ are illustrated below.

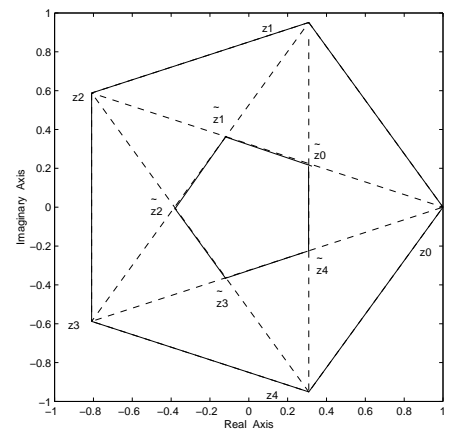

Lemma 2.7. Let $A \in \mathcal{M}_{n}(\mathbb{R})$ be nonnegative with irreducible hermitian part $H(A)$ such that $0<r_{j}(A) \in \Lambda_{j}\left(e^{-i \theta_{j}} A\right)$ for some $\theta_{j} \in[0,2 \pi)$ and every $j=1,2, \ldots, k$. If there exists an angle $\phi \in \mathbb{R}$ such that $r(A) e^{i \phi} \in F(A)$, then $r_{j}(A) e^{i \phi} \in \Lambda_{j}\left(e^{-i \theta_{j}} A\right)$ for every $j=1, \ldots, k$.

Proof. Suppose $r(A) e^{\mathbf{i} \phi} \in F(A)$ for some $\phi \in \mathbb{R}$, then by proposition 3.7 in 13 we have that $e^{-\mathbf{i} \phi} A=D^{-1} A D$ for some unitary diagonal matrix $D$. Obviously, $0<r_{j}(A) \in \Lambda_{j}\left(e^{-\mathbf{i} \theta_{j}} A\right)=\Lambda_{j}\left(e^{-\mathbf{i}\left(\theta_{j}+\phi\right)} A\right)$, therefore $r_{j}(A) e^{\mathbf{i} \phi} \in$ $\Lambda_{j}\left(e^{-\mathbf{i} \theta_{j}} A\right)$ for every $j=1, \ldots, k$.

Investigating $\Lambda_{k}(A)$ of a nonnegative matrix $A$ in terms of the irreducibility of the hermitian part $H(A)$ of the matrix $A$, we extend an analogous discussion developed in [13, 14].

Proposition 2.8. Let $A \in \mathcal{M}_{n}(\mathbb{R}), A \geq 0$ with $H(A)$ be irreducible and $r_{k}(A)>0$. Then either $\mathcal{F}_{j}(A)$ coincides with the circle $\mathcal{S}\left(0, r_{j}(A)\right)$ for every $j=1, \ldots, k$ or $\mathcal{F}_{j}(A)=\left\{r_{j}(A) e^{i\left(\theta_{j}+\frac{2 \pi t}{q}\right)}: t=0, \ldots, q-1\right\}$ for every $j=1, \ldots, k$, where $\theta_{j}=0$ or $\theta_{j}=\frac{\pi}{q}$ and $q>1$ is the largest positive integer such that $A$ is diagonally similar to the matrix $e^{i \frac{2 \pi}{q}} A$.

Proof. Suppose that for each $j=1, \ldots, k$ we have $0<r_{j}(A) \in \Lambda_{j}\left(e^{-\mathbf{i} \theta_{j}} A\right)$ for some principal argument $\theta_{j} \in[0,2 \pi)$. Due to proposition 3.11 in [13] 
and corollary 3.6 in [14], either $\mathcal{F}(A)=\mathcal{S}(0, r(A))$ or $\mathcal{F}(A)=\left\{r(A) e^{\mathbf{i} \frac{2 \pi t}{q}}\right.$ : $t=0, \ldots, q-1\}$, where $q$ is the maximum positive integer such that $A$ is unitarily diagonally similar to the matrix $e^{\mathbf{i} \frac{2 \pi}{q}} A$. The first equality of the sets indicates that $r(A) e^{\mathbf{i} \phi} \in F(A)$ for every angle $\phi \in \mathbb{R}$, thus by Lemma 2.7. $r_{j}(A) e^{\mathbf{i} \phi} \in \Lambda_{j}\left(e^{-\mathbf{i} \theta_{j}} A\right)$ for every angle $\phi \in \mathbb{R}$, concluding $\mathcal{F}_{j}(A)=$ $\mathcal{S}\left(0, r_{j}(A)\right)$ for all $j=1, \ldots, k$. For the second case where $r(A) e^{\mathbf{i} \frac{2 \pi t}{q}} \in F(A)$, Lemma 2.7 verifies $r_{j}(A) e^{\mathbf{i} \frac{2 \pi t}{q}} \in \Lambda_{j}\left(e^{-\mathbf{i} \theta_{j}} A\right)$ and then

$$
\left\{r_{j}(A) e^{\mathbf{i}\left(\theta_{j}+\frac{2 \pi t}{q}\right)}: t=0, \ldots, q-1\right\} \subseteq \mathcal{F}_{j}(A) .
$$

The equality of the sets, with $\theta_{j}=0$ or $\frac{\pi}{q}$ is established similarly as in the proof of Proposition 2.4, since $q$ is identified with the largest positive integer such that $e^{-\mathbf{i} \theta_{j}} A$ is unitarily diagonally similar to the matrix $e^{\mathbf{i}\left(-\theta_{j}+\frac{2 \pi}{q}\right)} A$ and $\Lambda_{j}(A)$ are symmetric with respect to the real axis for every $j=1, \ldots, k$.

Corollary 2.9. Let $A \in \mathcal{M}_{n}(\mathbb{R}), A \geq 0$ with irreducible hermitian part. If $0<r_{j}(A) \notin \Lambda_{j}(A)$ for some $j=2, \ldots, k$ and $\Lambda_{j}(A)$ is not a circular disc, then $\Lambda_{j}(A)$ is symmetric with respect to the lines $\mathcal{L}_{ \pm}=\left\{z e^{ \pm i \frac{\pi}{q}}: z \in \mathbb{R}\right\}$, where $q$ is the largest positive integer such that $A$ is diagonally similar to $e^{i \frac{2 \pi}{q}} A$.

Proof. By Proposition 2.8, $\mathcal{F}_{j}(A)=\left\{r_{j}(A) e^{\mathrm{i} \frac{\pi(2 t+1)}{q}}: t=0, \ldots, q-1\right\}$, whereupon

$$
\mathcal{F}_{j}\left(e^{ \pm \mathbf{i} \frac{\pi}{q}} A\right)=\left\{r_{j}(A) e^{\mathbf{i} \frac{2 \pi t}{q}}: t=0, \ldots, q-1\right\} \subseteq \partial \Lambda_{j}\left(e^{ \pm \mathbf{i} \frac{\pi}{q}} A\right),
$$

for some $j=2, \ldots, k$. Clearly, $\Lambda_{j}\left(e^{ \pm \mathbf{i} \frac{\pi}{q}} A\right)$ is symmetric with respect to $\mathbb{R}$, implying $\Lambda_{j}(A)=e^{\mp \mathbf{i} \frac{\pi}{q}} \Lambda_{j}\left(e^{ \pm \mathbf{i} \frac{\pi}{q}} A\right)$ to be symmetric with respect to the lines $\mathcal{L}_{ \pm}$

Corollary 2.10. Let $A \in \mathcal{M}_{n}(\mathbb{R}), A \geq 0$ with $H(A)$ be irreducible such that $r_{k}(A)>0$. If $F(A)$ is a circular disc, then $\Lambda_{j}(A)$ is also a circular disc for every $j=2, \ldots, k$.

Any matrix of the form (2.1) with $C_{q 1}=0$ and $q>1$ is called to be a block-shift matrix. It is already known that for real nonnegative matrices with irreducible hermitian part, which can be put into the block-shift form by means of a permutation, we obtain the circularity of the numerical range [16, Th.1]. Because of Corollary 2.10, it is immediate that this type of matrices also characterize the circularity of the higher rank numerical range.

Proposition 2.11. Let $A \in \mathcal{M}_{n}(\mathbb{R}), A \geq 0$ with $H(A)$ be irreducible. If $A$ is permutation similar to a block-shift matrix, then $\Lambda_{j}(A)$ is identified with the circular disc $\mathcal{D}\left(0, r_{j}(A)\right)$ for $j=1, \ldots, k$. 
We should note that the conclusion of the preceding proposition does not hold in general for any complex matrix $A$ being unitarily similar to a block-shift matrix. For the general case, we present the next result.

Proposition 2.12. Let $A \in \mathcal{M}_{n}(\mathbb{C})$ be unitarily similar to $\left[\begin{array}{cc}0 & A_{1} \\ 0 & 0\end{array}\right]$ with $A_{1} \in \mathcal{M}_{m, n-m}(\mathbb{C})$ and $\operatorname{rank} A_{1}=k$. Then

$$
\Lambda_{j}(A)=\mathcal{D}\left(0, \frac{\sigma_{j}\left(A_{1}\right)}{2}\right) \text { for } j=1, \ldots, k,
$$

where $\sigma_{j}\left(A_{1}\right)$ denotes the $j$-th largest singular value of $A_{1}$ and then $r_{j}(A)=$ $\frac{\sigma_{j}\left(A_{1}\right)}{2}$, for $j=1, \ldots, k$.

Proof. It only suffices to prove that $\Lambda_{j}\left(\left[\begin{array}{cc}0 & A_{1} \\ 0 & 0\end{array}\right]\right)=\mathcal{D}\left(0, \frac{\sigma_{j}\left(A_{1}\right)}{2}\right)$, for $j=$ $1, \ldots, k$, since $\Lambda_{j}(A)$ is invariant under unitary equivalence. Therefore, by 12]

$$
\Lambda_{j}\left(\left[\begin{array}{cc}
0 & A_{1} \\
0 & 0
\end{array}\right]\right)=\bigcap_{\theta \in[0,2 \pi)} e^{-\mathbf{i} \theta}\left\{z \in \mathbb{C}: \operatorname{Re} z \leq \lambda_{j}\left(H\left(\left[\begin{array}{cc}
0 & e^{\mathbf{i} \theta} A_{1} \\
0 & 0
\end{array}\right]\right)\right)\right\},
$$

where $\lambda_{j}(H(A))$ denotes the $j$-th largest eigenvalue of the hermitian part $H(A)$ of matrix $A$. Hence

$$
\Lambda_{j}\left(\left[\begin{array}{cc}
0 & A_{1} \\
0 & 0
\end{array}\right]\right)=\bigcap_{\theta \in[0,2 \pi)} e^{-\mathbf{i} \theta}\left\{z \in \mathbb{C}: \operatorname{Re} z \leq \frac{1}{2} \lambda_{j}\left(\left[\begin{array}{cc}
0 & e^{\mathbf{i} \theta} A_{1} \\
e^{-\mathbf{i} \theta} A_{1}^{*} & 0
\end{array}\right]\right)\right\} .
$$

It is known that the eigenvalues of the hermitian matrix $\left[\begin{array}{cc}0 & e^{\mathbf{i} \theta} A_{1} \\ e^{-\mathbf{i} \theta} A_{1}^{*} & 0\end{array}\right]$ are the singular values $\sigma_{1}\left(A_{1}\right) \geq \ldots \geq \sigma_{k}\left(A_{1}\right)>0 \geq \ldots \geq 0>-\sigma_{k}\left(A_{1}\right) \geq$ $\ldots \geq-\sigma_{1}\left(A_{1}\right)$ with $k=\operatorname{rank} A_{1}[$ ], thus for $j=1, \ldots, k$

$$
\Lambda_{j}\left(\left[\begin{array}{cc}
0 & A_{1} \\
0 & 0
\end{array}\right]\right)=\bigcap_{\theta \in[0,2 \pi)} e^{-\mathbf{i} \theta}\left\{z \in \mathbb{C}: \operatorname{Re} z \leq \frac{\sigma_{j}\left(A_{1}\right)}{2}\right\}=\mathcal{D}\left(0, \frac{\sigma_{j}\left(A_{1}\right)}{2}\right) .
$$

\section{Application to matrix polynomials}

An extension of the aforementioned results to the higher rank numerical range of a matrix polynomial arises naturally and it is the purpose of this section. For this reason, we refer to Perron polynomials $L(\lambda)$, which are $n \times n$ monic matrix polynomials of $m$ th degree

$$
L(\lambda)=I \lambda^{m}-A_{m-1} \lambda^{m-1}-\ldots-A_{1} \lambda-A_{0},
$$


with $A_{j}$ to be nonnegative matrices, for $j=0, \ldots, m-1$. The higher rank numerical range $\Lambda_{k}(L(\lambda))$ of $L(\lambda)$ has been recently defined in [1] by the set

(3.2) $\Lambda_{k}(L(\lambda))=\left\{\lambda \in \mathbb{C}: Q^{*} L(\lambda) Q=0_{k}\right.$ for some $\left.Q \in \mathcal{M}_{n, k}, Q^{*} Q=I_{k}\right\}$,

which for $k=1$ yields the numerical range

$$
w(L(\lambda))=\left\{\lambda \in \mathbb{C}: x^{*} L(\lambda) x=0 \text { for some } x \in \mathbb{C}^{n}, x^{*} x=1\right\} .
$$

The notion of the Perron polynomial $L(\lambda)$ in (3.1) is equivalent to the nonnegativity of its $m n \times m n$ companion matrix

$$
C_{L}=\left[\begin{array}{ccccc}
0 & I_{n} & 0 & \cdots & 0 \\
0 & 0 & I_{n} & \cdots & 0 \\
\vdots & & \ddots & \ddots & \vdots \\
0 & & & & I_{n} \\
A_{0} & & \cdots & & A_{m-1}
\end{array}\right]
$$

hence an extension of the main Perron-Frobenius theorem concerning the spectrum $\sigma(L)$ of $L(\lambda)$ and Issos' results on the numerical range $w(L(\lambda))$ are established via the companion matrix $C_{L}$ [15]. Further, we denote the rank $k$-numerical radius of $\Lambda_{k}(L(\lambda))$

$$
r_{k}(L)=\max \left\{|\lambda|: \lambda \in \Lambda_{k}(L(\lambda))\right\},
$$

with $r_{k}(L)=-\infty$ when $\Lambda_{k}(L(\lambda))=\emptyset$ and consequently, the set of maximal elements in $\Lambda_{k}(L(\lambda))$ :

$$
\mathcal{F}_{k}(L)=\left\{\lambda \in \Lambda_{k}(L(\lambda)):|\lambda|=r_{k}(L)\right\} .
$$

As we have noticed in section 2 , clearly $\Lambda_{k}(L(\lambda))$ does not always contain the element $r_{k}(L)>0$.

The following lemma is a generalization of a corresponding result in [15], which identifies $\Lambda_{k}(L(\lambda))$ with a specific subset of $\Lambda_{k}\left(C_{L}\right)$ of the companion matrix $C_{L}$ of a matrix polynomial $L(\lambda)$. We should recall that $\Lambda_{k}\left(C_{L}\right)$ always contains $\Lambda_{k}(L(\lambda))$ as it has been proved in [1, Prop.16].

Lemma 3.1. Let the $m n \times k$ matrix $Y(\lambda, Q)=\left(1, \lambda, \ldots, \lambda^{m-1}\right)^{T} \otimes Q$ with $\lambda \in \mathbb{C}$ and $Q \in \mathcal{M}_{n, k}$. Then

$$
\begin{aligned}
& \Lambda_{k}(L(\lambda)) \cup\{0\}=\left\{\lambda: Y^{*}(\lambda, Q) C_{L} Y(\lambda, Q)=\lambda I_{k}, Q \in \mathcal{M}_{n, k}, Q^{*} Q=\frac{1}{c\left(|\lambda|^{2}\right)} I_{k}\right\}, \\
& \text { where } c\left(|\lambda|^{2}\right)=\left(1+|\lambda|^{2}+|\lambda|^{4}+\ldots+|\lambda|^{2(m-1)}\right)^{1 / 2} .
\end{aligned}
$$


Proof. It is readily verified that

$$
Y^{*}(\lambda, Q)\left(C_{L}-\lambda I_{m n}\right) Y(\lambda, Q)=\bar{\lambda}^{m-1} Q^{*} L(\lambda) Q
$$

and $Y^{*}(\lambda, Q) Y(\lambda, Q)=c\left(|\lambda|^{2}\right) Q^{*} Q$. Hence, $\lambda_{0} \in \Lambda_{k}(L(\lambda))$ if and only if $\sqrt{c\left(\left|\lambda_{0}\right|^{2}\right)} Q^{*} L\left(\lambda_{0}\right) Q \sqrt{c\left(\left|\lambda_{0}\right|^{2}\right)}=0_{k}$ and equivalently when $Q^{*} L\left(\lambda_{0}\right) Q=0_{k}$.

Proposition 3.2. Let $L(\lambda)$ be a Perron polynomial as in (3.1) such that $r_{k}(L)>0$, with irreducible companion matrix $C_{L}$ and $q>1$ eigenvalues of maximum modulus. Then the maximal elements in $\Lambda_{j}(L(\lambda))$ are exactly the set

$$
\mathcal{F}_{j}(L)=\left\{r_{j}(L) e^{i\left(\theta_{j}+\frac{2 \pi t}{q}\right)}, t=0, \ldots, q-1\right\},
$$

where $\theta_{j}=0$ or $\theta_{j}=\frac{\pi}{q}$, for $j=1, \ldots, k$.

Proof. The companion matrix $C_{L} \geq 0$ is an imprimitive matrix with index of imprimitivity $q>1$, since $\sigma(L)=\sigma\left(C_{L}\right)$. Hence $q$ is the largest positive integer such that $C_{L}$ is unitarily diagonal to $e^{\mathrm{i} \frac{2 \pi}{q}} C_{L}$. Therefore, $e^{\mathrm{i} \frac{2 \pi t}{q}} C_{L}=D^{-1} C_{L} D(t=0,1, \ldots, q-1)$, for some unitary diagonal $m n \times m n$ matrix $D=\operatorname{diag}\left(D_{0}, e^{\mathbf{i} \frac{2 \pi t}{q}} D_{0}, \ldots, e^{\mathbf{i} \frac{2 \pi t(m-1)}{q}} D_{0}\right)$, with $D_{0} \in \mathcal{M}_{n}$ to be unitary diagonal. Suppose $\theta_{j} \in[0,2 \pi)$ is the principal argument such that $0<r_{j}(L) \in \Lambda_{j}\left(e^{-\mathbf{i} \theta_{j}} L(\lambda)\right)$, then by Lemma 3.1 we have

$$
\begin{aligned}
0_{k} & =Y^{*}\left(r_{j}(L) e^{\mathbf{i} \theta_{j}}, Q\right)\left(C_{L}-r_{j}(L) e^{\mathbf{i} \theta_{j}} I_{m n}\right) Y\left(r_{j}(L) e^{\mathbf{i} \theta_{j}}, Q\right) \\
& =Y^{*}\left(r_{j}(L) e^{\mathbf{i} \theta_{j}}, Q\right) D^{-1}\left(e^{-\mathbf{i} \frac{2 \pi t}{q}} C_{L}-r_{j}(L) e^{\mathbf{i} \theta_{j}} I_{m n}\right) D Y\left(r_{j}(L) e^{\mathbf{i} \theta_{j}}, Q\right) \\
& =Y^{*}\left(r_{j}(L) e^{\mathbf{i}\left(\theta_{j}+\frac{2 \pi t}{q}\right)}, D_{0} Q\right)\left(C_{L}-r_{j}(L) e^{\mathbf{i}\left(\theta_{j}+\frac{2 \pi t}{q}\right)} I_{m n}\right) Y\left(r_{j}(L) e^{\mathbf{i}\left(\theta_{j}+\frac{2 \pi t}{q}\right)}, D_{0} Q\right),
\end{aligned}
$$

with $Y^{*}\left(e^{\mathbf{i} \frac{2 \pi t}{q}} \lambda, D_{0} Q\right) Y\left(e^{\mathbf{i} \frac{2 \pi t}{q}} \lambda, D_{0} Q\right)=Y^{*}(\lambda, Q) Y(\lambda, Q)=I_{m n, k}$ for any scalar $\lambda$. Apparently,

$$
\mathcal{F}_{j}(L) \supseteq\left\{r_{j}(L) e^{\mathbf{i}\left(\theta_{j}+\frac{2 \pi t}{q}\right)}, t=0, \ldots, q-1\right\} .
$$

Further, as in the proof of Proposition 2.4, we establish the equality of the sets with $\theta_{j}=0$ or $\theta_{j}=\frac{\pi}{q}$ for $j=1, \ldots, k$, since the coefficients of $L(\lambda)$ are real, whereupon $\Lambda_{k}(L(\lambda))$ is symmetric with respect to the real axis.

\section{References}

[1] Aik. Aretaki and J. Maroulas, The higher rank numerical range of matrix polynomials, 10th Workshop on the "Numerical Range and Numerical Radii", Krakow, Poland, 2010, submitted for publication. 
[2] M.D. Choi, M. Giesinger, J.A. Holbrook and D.W. Kribs, Geometry of higher-rank numerical ranges, Linear and Multilinear Algebra, 56(1), 53-64, 2008.

[3] M.D. Choi, J.A. Holbrook, D.W. Kribs and K. Zyczkowski, Higherrank numerical ranges of unitary and normal matrices, preprint, http://arxiv.org/quant-ph/0608244.

[4] M.D. Choi, D.W. Kribs and K. Zyczkowski, Quantum error correcting codes from the compression formalism, Reports on Mathematical Physics, 58, 77-86, 2006.

[5] M.D. Choi, D.W. Kribs and K. Zyczkowski, Higher-rank numerical ranges and compression problems, Linear Algebra and its Applications, 418, 828-839, 2006.

[6] H.L. Gau, C.K. Li, Y.T. Poon and N.S. Sze, Quantum error correction and higher rank numerical ranges of normal matrices, preprint, http://arxiv.org/0902.4869v1 [math.FA], 2009, SIAM J. Matrix Analysis and Applications, (to appear).

[7] K.E. Gustafson and D.K.M. Rao, Numerical Range. The Field of Values of Linear Operators and Matrices, Springer-Verlag, New York, 1997.

[8] R.A. Horn and C.R. Johnson, Matrix Analysis, Cambridge University Press, Cambridge, 1985.

[9] R.A. Horn and C.R. Johnson, Topics in Matrix Analysis, Cambridge University Press, Cambridge, 1991.

[10] J.N. Issos, The field of values of non-negative irreducible matrices, Ph.D. Thesis, Auburn University, 1966.

[11] C.K. Li, Y.T. Poon and N.S. Sze, Condition for the higher rank numerical range to be non-empty, Linear and Multilinear Algebra, 57(4), 365-368, 2009.

[12] C.K. Li and N.S. Sze, Canonical forms, higher rank numerical ranges, totally isotropic subspaces, and matrix equations, Proceedings of the American Mathematical Society, 136, 3013-3023, 2008.

[13] C.K. Li, B.S. Tam and P.Y. Wu, The numerical range of a nonnegative matrix, Linear Algebra and its Applications, 350, 1-23, 2002.

[14] J. Maroulas, P.J. Psarrakos and M.J Tsatsomeros, Perron-Frobenius type results on the numerical range, Linear Algebra and its Applications, 348, 49-62, 2002. 
[15] P.J. Psarrakos and M.J Tsatsomeros, A primer of Perron-Frobenius theory for matrix polynomials, Linear Algebra and its Applications, 393, 333-351, 2004.

[16] B.S. Tam and S. Yang. On matrices whose numerical ranges have circular or weak circular symmetry, Linear Algebra and its Applications, 302-303, 193-221, 1999.

[17] H.J. Woerdeman, The higher rank numerical range is convex, Linear and Multilinear Algebra, 56(1), 65-67, 2007. 